Author(s):

Title:

Night Vision Device Technology Development

Submitted to:

Herbert Funsten, NIS-1

Jane Nordholt, NIS-1

David Suszcynsky, NIS-1

DOE Office of Scientific and Technical Information (OSTI)
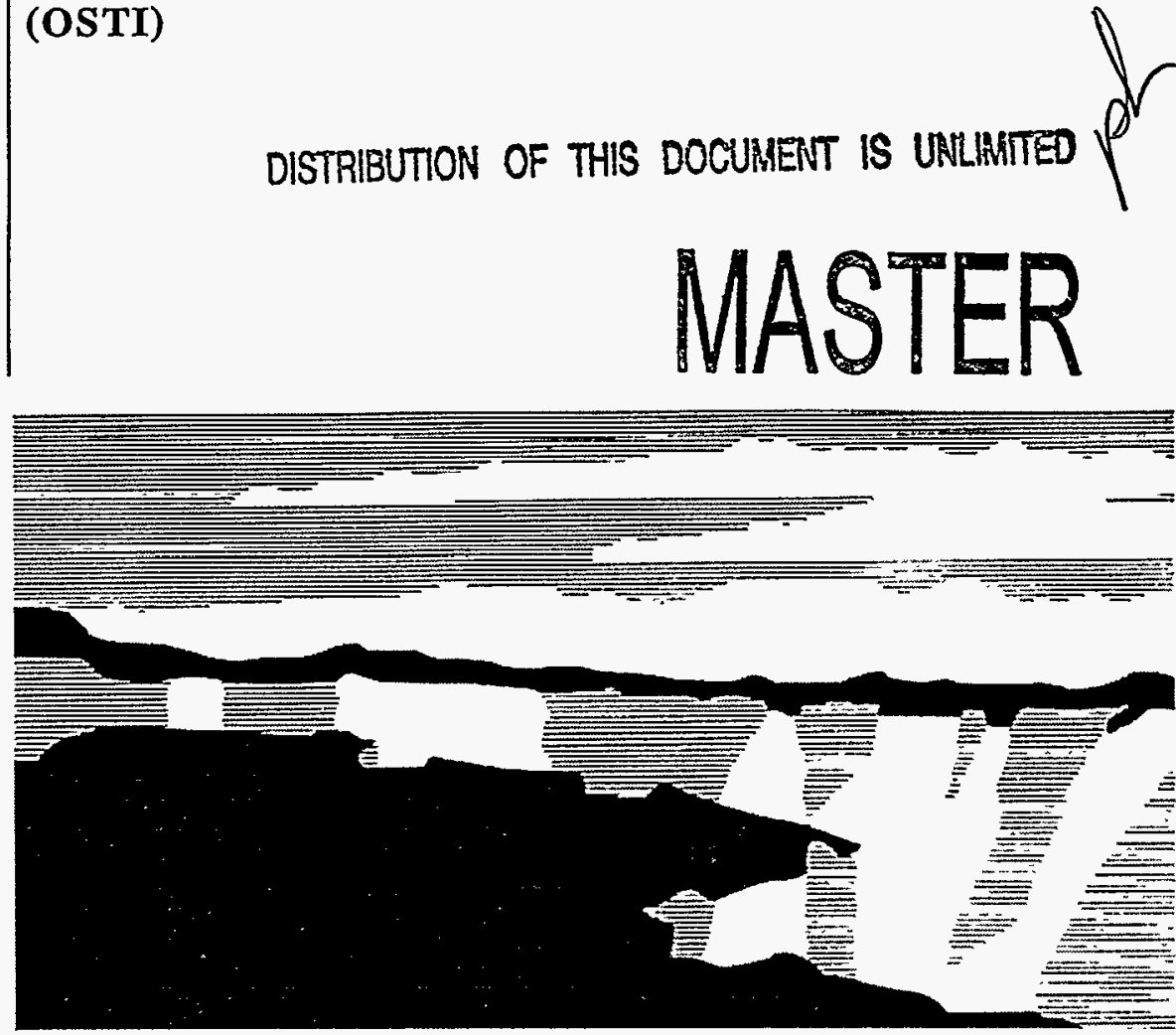

NATIONAL LABORATORY

Los Alamos National Laboratory, an affirmative action/equal opportunity employer, is operated by the University of California for the U.S. Department of Energy under contract W-7405-ENG-36. By acceptance of this article, the publisher recognizes that the U.S. Government retains a nonexclusive, royaltyfree license to publlsh or reproduce the published form of this contribution, or to allow others to do so, for U.S. Government purposes. The Los Alamos Natlonal Laboratory requests that the publisher ldentify this article as work performed under the auspices of the U.S. Department of Energy. 


\section{DISCLAIMER}

This report was prepared as an account of work sponsored by an agency of the United States Government. Neither the United States Government nor any agency thereof, nor any of their employees, makes any warranty, express or implied, or assumes any legal liability or responsibility for the accuracy, completeness, or usefulness of any information, apparatus, product, or process disclosed, or represents that its use would not infringe privately owned rights. Reference herein to any specific commercial product, process, or service by trade name, trademark, manufacturer, or otherwise does not necessarily constitute or imply its endorsement, recommendation, or favoring by the United States Government or any agency thereof. The views and opinions of authors expressed herein do not necessarily state or reflect those of the United States Government or any agency thereof. 


\section{DISCLAIMER}

Portions of this document may be illegible in electronic image products. Images are produced from the best available original document. 


\title{
Night Vision Device Technology Development
}

\author{
Herbert Funsten*, Jane Nordholt, and David Suszcynsky
}

\begin{abstract}
This is the final report of a one-year, Laboratory-Directed Research and Development (LDRD) project at the Los Alamos National Laboratory (LANL). This project sought to develop microchannel plate (MCP) technologies for enhancement of night vision device (NVD) capabilities. First, segmented microchannel plates with independent gain control to minimize loss of low level light images in the presence of a bright light source (e.g., battlefield lasers, flares, and headlights) need to be developed. This enables, for example, enhanced vision capabilities during night operations in, for example, a city environment and continuous capability of aviators to see the horizon, nearground obstructions, and ground targets. Furthermore, curved microchannel plate technology to increase the field of view of NVDs while minimizing optical aberrations needs to be developed and applied. This development would significantly enhance peripheral vision capabilities of aviators and result in easier adaptation of the human eye to NVDs.
\end{abstract}

\section{Background and Research Objectives}

Night vision devices (NVDs) utilizing image intensification tubes are employed for assisted viewing under low-light-level conditions in a variety of night operations including suspect location and apprehension, targeting, aircraft piloting, surveillance, and battlefield viewing. The two basic types of NVD are image intensifiers (on which we focus here) and thermal detectors. Image intensifier NVDs typically provide better spatial resolution, broader band wavelength sensitivity, and greater portability. They operate by converting photons to electrons, multiplying the electrons by microchannel plates (MCPs), and converting the electrons back to photons. Several versions of this type of NVD are standard issue in the military.

* Principal investigator, e-mail: hfunsten@lanl.gov 
We have identified two significant limitations in NVDs currently utilized by law enforcement and the military: (1) loss of low-light-level images in the presence of a bright light source shining at the NVDs and (2) a small field of view that is restricted by optical aberrations introduced by a flat image plane in the image intensification tube.

A bright light shining at the NVD (e.g., headlight, streetlight, or laser) triggers an electronic circuit, called the automatic gain control (AGC), that reduces the light amplification in the NVD so that the microchannel plates in NVD image intensifier tubes will not be damaged. This results in a failure to see very dim objects with a bright light source in the NVD field of view. For example, a targeting laser, streetlight, flare, or other bright light source observed by an aviator using a NVD may no longer see the dim horizon (which is critical for navigation), near-ground obstructions with which his aircraft may collide, or targets/activities on the ground. For suspect location or night operations in a city environment, bright lights such as street lights and headlights may significantly impede ones ability to view adjacent, dimly lit areas.

The critical problem of reduced light amplification in a NVD when a bright light source is in the field of view can be overcome by segmentation of the MCPs into different electronic regions and utilizing an independent AGC circuit for each segment. Therefore, a bright light source in one segment within the field of view will reduce the gain in that segment only, so that all other segments retain high gain and enable viewing of dim background objects in a large majority of the field of view. This enhances vision capabilities such as observing a dim background in a city setting having bright lights (e.g., streetlights, headlights) shining into the NVD. This can be applied to a wide variety of night operations such as those operations in a city environment. Furthermore, it enables aviators to see the horizon, near-ground obstructions, and dim ground activities in a field of view having bright light sources. For this part of the project, we intent to develop, fabricate, and test a segmented MCP detector array with individual AGCs for each segment. MCP segmenting can be performed by advanced manufacturing such as laser-milling or selective-area deposition (i.e., lithography).

The second limitation of NVDs involves their restricted field of view (FOV). Current NVDs utilized by the military have a maximum FOV of $40^{\circ} \times 40^{\circ}$ that is inherently limited due to optical aberrations introduced by the flat MCPs located at the focal plane of the image intensification tube. This restricted FOV is significantly less than that of the human eye $\left(120^{\circ}\right.$ $x 150^{\circ}$ ); in fact, both eyes together can provide a horizontal FOV of as much as $200^{\circ}$. This disparity results in difficult adaptation of the human eye to NVD use (especially by aviators), and the lack of peripheral vision motion cues is a major cause of nighttime military aircraft accidents when these devices are used. 
Recent MCP technology developments have resulted in fabrication of curved (domed) MCPs (these curved MCPs are currently used in the x-ray telescopes on the ALEXIS spacecraft). Applying curved MCPs to night vision devices can yield a considerably wider FOV while inducing minimal optical aberrations. These curved MCPs can provide a "bugeyed" FOV that more closely matches that of the human eye. Furthermore, gain balancing of MCPs can also be employed to produce NVDs with separate MCP stacks for each eye. This is typically not utilized in current NVDs due to unusual three-dimensional effects that occur if the MCP gains (light amplification) are not exactly balanced.

The goal of this project was to develop and apply curved microchannel plate technology to increase the field of view of NVDs while minimizing optical aberrations to enable peripheral vision capabilities to NVD users and easier adaptation of the human eye to NVDs. This technology is particularly applicable to aviators, since a majority of nighttime collisions are due to restricted vision capabilities. Development of this technology can significantly extend the surveillance field of view and reduce the associated eye fatigue; for aviators, this technology would provide a significantly safer environment with enhanced piloting capabilities.

\section{Importance to LANL's Science and Technology Base and National R\&D Needs}

This project entails basic development of an advanced sensor. Advanced sensors are one component of the Los Alamos core competency in complex experimentation and measurement. This project involves integrated research in which detector technologies for space-flight applications are being applied to ground-based, night-vision device enhancement. The goal of this project was to confirm proof-of-principle of the NVD development concepts and initiate collaborative efforts with industry for full development, testing, and integration of demonstration NVDs based on these concepts.

\section{Scientific Approach and Results to Date}

The enhancement of viewing dim objects near a bright light source will provide a clear advantage to the NVD user. Using existing NVD technology, a bright light source of 0.01 foot candles (e.g., a headlight shining at the NVD user) will suppress NVD output brightness of a dim background image of 0.0001 foot candles (e.g., a hostile target) by a factor of 100 so that the dim image is not observable using a NVD. In this case, if the bright light source is not present then the dim image is 100 times brighter than if the bright light source was present. By segmenting the MCP in the NVD, the bright light source will affect only a small portion of the field of view (segment size is targeted to be $5 \%$ of the total field of view), so that dim images 
in the background remain bright in the NVD. The modified NVD should fit into existing NVD hardware.

Segmented MCP development tasks on this project included the following: (1) use of particle simulations to develop detailed design for MCP segmentation and use these results to minimize "dead area" between segments and (2) obtaining MCP with and without inconel on surfaces, examination of methods to manufacture MCPs with selected-area inconel or $\mathrm{Ni}$ coatings, selective area deposition by evaporating conductor on MCPs using masks, electronbeam or photo-lithography techniques, and laser milling of conductor from surface.

The wide field of view NVDs that are developed should have at least a $60^{\circ} \times 60^{\circ}$ field of view for each eye, providing a total $90^{\circ}$ (horizontal) $\times 60^{\circ}$ (vertical) field of view when used in tandem. This is more than three times the field of view of current NVDs, and would provide minimal aberrations and eye strain to the user. Furthermore, a user would observe a wide field of view with minimal head motion, reducing neck strain when used for extended periods. Curved MCPs are required for wide field of view NVGs.

Curved MCP development tasks on this project included the following: (1) development of detailed optical and electro-optical design to obtain widest FOV using currently available curved MCPs and utilization of ray-tracing codes to optimize design and (2) procurement, fabrication, and testing of components as necessary.

Night vision devices utilizing image intensification tubes, used extensively by the US military, have two significant problems that can critically compromise their operation: the field of view is restricted due to abberations introduced by the flat image plane and gain (light sensitivity) loss when a bright light source is within the field of view. A wide field of view is critical to aviators, who need peripheral vision for motion cues and nearby obstacles/ obstructions/aircraft, and the bright spot suppression is crucial to night missions performed in an urban or well-lighted environment (streetlights, flares, headlights).

We have developed two technologies to overcome these problems. First, we have designed a curved image plane using recently-developed detector technology for the wide field of view. Optics and electro-optical simulations have been performed to enable up to 80 degrees field-of-view. For the bright spot suppression, we have designed segmented microchannel plates and the associated independent gain control circuitry to suppress the gain in the segment illuminated by a bright light source while retaining full gain across all other segments, so that dim objects within the field of view remain visible. Furthermore, we have initiated a collaborative effort with an industrial partner to develop the proof-of-principle prototypes to fruition and to evaluate the possibility of retrofitting the technologies into existing night vision devices. 